\title{
Analysis of Filtering Effect and Energy Saving Mechanism of Dynamic Harmonic Filter
}

\author{
Wang Yifei ${ }^{1, a}$, Yuan Youxin ${ }^{2, a}$, Mao Jinming ${ }^{3, b}$ and Jiang Yongming ${ }^{4, c}$ \\ ${ }^{1}$ School of Automation, Wuhan University of Technology, Wuhan 430070, China \\ ${ }^{2}$ Nanjing Kangdixin Electric Unit LtD., Nanjing 210002, China \\ ${ }^{3}$ Changxin South Cement Company Limited, Huzhou, 313117, China \\ awyfnhsz88@163.com, byyx2000@263.net, ${ }^{c}$ 2851938043@qq.com
}

\begin{abstract}
Keywords: dynamic harmonic filter; harmonic loss; drift of the filter resonance point; power saving Abstract. The harmonic suppression must be applied to the power equipment in distribution system in order to improve the power quality and saving energy. So the author developed the dynamic harmonic filter, and it was successfully applied. Based on the previous research, Firstly, the paper analyzed quantitatively the harmonic loss of the power equipment in power distribution system and the drift of the filter resonance point caused by the change of the capacitance of the capacitor. Secondly, the paper discussed the energy saving mechanism of the dynamic harmonic filter, and developed a topology structure of magnetically controlled reactor, analyzed the adjustment principle of the inductance (L). Lastly, application analysis of engineering has been done. The application results show that the dynamic harmonic filter can reduce harmonic current, and can reduce the $5 \% \sim 10 \%$ fundamental current. So that direct power saving effect is more than 5\%, and the copper loss and iron loss is reduced by $3 \%$. The total energy saved in the system reaches more than $10 \%$.
\end{abstract}

\section{Introduction}

China's electric power industry is developing rapidly. There are industrial power equipment, such as cement mill and kiln, electrified rail, rolling mill, and mine hoist. A large number of reactive powers will be consumed when the equipment is running, and their speed regulating device can produce a large number of harmonics. It leads to energy consumption, and reduces the power quality. Therefore, in order to improve the quality of power grid, better energy saving, harmonic suppression and reactive power compensation is particularly important [1]- [3].

For above reason, the author developed the dynamic harmonic filter [4], and it was successfully applied. Based on the previous research, the paper analyzes quantitatively the harmonic loss of the power equipment in power distribution system and the drift of the filter resonance point caused by the change of the capacitance of the capacitor, and then discusses the energy saving mechanism of the dynamic harmonic filter and advances the method for keeping filtering effect of the dynamic harmonic filter. Finally, the filtering effect and energy saving effect of the dynamic harmonic filtering energy saving device is verified by the engineering application.

\section{Harmonic Loss Analysis of Power Equipment in Distribution System}

In the case of the presence of harmonic distortion, the total loss can be expressed as the sum of the fundamental wave and the losses at each harmonic frequency, it is as follow:

$$
P=P_{1}+\sum_{h=2}^{n} P_{h}
$$

Where, $P_{1}$ is losses of the fundamental wave, $P_{h}$ is (h value is $2,3, \ldots, \mathrm{n}$ ) the losses at $\mathrm{h}$ order harmonic frequency. 
The harmonic losses of power equipment in distribution system mainly include the loss of harmonic generation during the operation of power equipment and the loss of the harmonic generation during the operation of the transformer.

Loss of Harmonic Generation during the Operation of Power Equipment. The loss of harmonic generation during the operation of power equipment is related to additional heat. The harmonic current increases the effective value of the current, and the value is calculated as follows:

$$
\mathrm{I}_{\mathrm{rms}}=\sqrt{\mathrm{I}_{1}^{2}+\sum_{\mathrm{h}=2}^{\mathrm{n}} \mathrm{I}_{\mathrm{h}}^{2}}
$$

Where, $I_{1}$ is the current of the fundamental wave, $I_{h}$ is (h value is $2,3, \ldots, \mathrm{n}$ ) the current at $\mathrm{h}$ order harmonic frequency. Therefore, the effective value of the current can be reduced by reducing the harmonic current.

The active power, reactive power and apparent power are as follow:

$$
\left\{\begin{array}{l}
P=\frac{1}{T} \int_{0}^{T} p(t) d t=\sum_{h=1}^{n} U_{h} I_{h} \cos \left(Q_{h}-\delta_{h}\right)=P_{1}+\sum_{h=2}^{n} P_{h} \\
Q=\frac{1}{T} \int_{0}^{T} q(t) d t=\sum_{h=1}^{\infty} U_{h} I_{h} \sin \left(Q_{h}-\delta_{h}\right)=Q_{1}+\sum_{h=2}^{\infty} Q_{h} \\
S=U_{r m s} I_{r m s}
\end{array}\right.
$$

Therefore, the harmonic power loss of the resistance $\left(\Delta \mathrm{P}_{\mathrm{R}}\right)$ and one of inductance elements $\left(\Delta \mathrm{P}_{\mathrm{L}}\right)$ are as follow:

$$
\left\{\begin{array}{c}
\Delta \mathrm{P}_{\mathrm{R}}=\mathrm{I}_{\mathrm{rms}}^{2} \mathrm{R}-\mathrm{I}_{1}^{2} \mathrm{R}=\left(\mathrm{I}_{\mathrm{rms}}^{2}-\mathrm{I}_{1}^{2}\right) \mathrm{R}=\sum_{\mathrm{h}=2}^{\mathrm{n}} \mathrm{I}_{\mathrm{h}}^{2} \mathrm{R} \\
\Delta \mathrm{P}_{\mathrm{L}}=\mathrm{I}_{\mathrm{rms}}^{2} \mathrm{X}_{\mathrm{L}}-\mathrm{I}_{1}^{2} \mathrm{X}_{\mathrm{L}}=\left(\mathrm{I}_{\mathrm{rms}}^{2}-\mathrm{I}_{1}^{2}\right) \mathrm{X}_{\mathrm{L}}=\sum_{\mathrm{h}=2}^{\mathrm{n}} \mathrm{I}_{\mathrm{h}}^{2} \mathrm{X}_{\mathrm{L}}
\end{array}\right.
$$

The harmonic power loss of the power equipment (Resistance and inductance components) $\Delta P_{Z}$ is as follow:

$$
\Delta \mathrm{P}_{\mathrm{Z}}=\sum_{\mathrm{h}=2}^{\mathrm{n}} \mathrm{I}_{\mathrm{h}}^{2} \mathrm{Z}
$$

The following conclusions can be drawn from the formula (5): Harmonic power loss can be reduced by reducing harmonics. ${ }^{\Delta \mathrm{P}_{2}}$ can fluctuate when load impedance change.

Loss of Harmonic Generation during the Operation of Transformer. The copper loss of the transformer winding is a function of the resistance (R0) at $50 \mathrm{~Hz}$ frequency. The resistance at $h$ order harmonic frequency is more than R0. It leads to increase in copper loss with added heat. Magnetizing current can be increased by the harmonic current. It leads to increase the core loss of AC iron core coil at with added heat.

In conclusion, the energy saving mechanism of the dynamic harmonic filter is as follow: as long as the harmonic current can be reduced, the active power consumed by the power equipment (nonlinear load) can be reduced. At the same time, it can reduce the loss of harmonic generation during the operation of the transformer. This has reached the purpose of energy saving. 


\section{Filter Resonance Point Drift of Caused by Capacitance Change}

At the harmonic frequency, the capacity capacitor $Q_{C}$ decreases with the increase of the time of use. It can result in resonant frequency shift, and educe harmonic filtering effect.

Taking the5th harmonic as an example: When the capacity $Q_{C}$ of the capacitor is reduced by $4 \%$, the capacitive reactance $X_{C}{ }^{\prime}$ is as follow:

$$
\mathrm{X}_{\mathrm{C}}{ }^{\prime}=\frac{\mathrm{U}_{\mathrm{C}}{ }^{2}}{(100-4) \% Q_{C}}=\frac{100}{96} \mathrm{X}_{\mathrm{C}}
$$

The capacitance value $C^{\prime}$ is as follow:

$$
C^{\prime}=\frac{1}{2 \pi f X_{C}{ }^{\prime}}=0.96 C
$$

So the 5th harmonic frequency is changed from $250 \mathrm{~Hz}$ to ${ }^{f_{5}}{ }^{\prime}$, its value is as follow:

$$
\mathrm{f}_{5}^{\prime}=\frac{1}{2 \pi \sqrt{\mathrm{LC}}}=\frac{1}{2 \pi \sqrt{\mathrm{LC}}} \sqrt{\mathrm{C} / \mathrm{C}^{\prime}}=\sqrt{1 / 0.96} \cdot \mathrm{f}_{5}=255 \mathrm{~Hz}
$$

The following conclusions can be drawn from the formula (8): the method for keeping filtering effect of the dynamic harmonic filter: When the capacity capacitor $Q_{c}$ is reduced, the capacitance value $\mathrm{C}$ becomes smaller. At this point, as long as the dynamic adjustment of the inductor $\mathrm{L}$, so that the product of $\mathrm{L}$ and $\mathrm{C}$ remains unchanged, that is the wave frequency $\mathrm{f}$ will not change. It can maintain the dynamic harmonic filter of the harmonic filtering effect.

For this purpose, the authors developed a magnetically controlled reactor (or MCR for short), to achieve the inductance of the $\mathrm{L}$ can be adjusted, but also to achieve dynamic harmonic filtering energy saving method of the core components.

\section{Topology Structure of MCR and its Inductance Adjustment Principle}

MCR is mainly composed of a magnetic controlled reactance converter (or MCRC for short) and a power electronic reactance converter (or PERC for short). The main control winding of the magnetic controlled reactance converter is connected with a power electronic reactance converter (anti parallel thyristor). The Topology structure of MCR is shown in figure 1.

The inductance adjustment principle of MCR: when

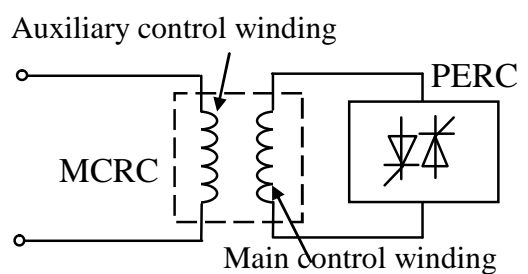

Fig. 1 Topology structure of change the phase control angle of thyristors, the impedance of the main control winding of the magnetic controlled reactance converter. The impedance of the auxiliary control winding is also changed by the electromagnetic coupling.

The relationship between the equivalent inductance $\mathrm{L}$ of auxiliary control winding and the thyristor controlled angle $\alpha[6]$ is as follow:

$$
L=\frac{\pi k^{2} L_{1}}{\sqrt{\sin ^{2} \alpha+(\pi-\alpha) \sin 2 \alpha+(\pi-\alpha)^{2}}}
$$

Where, $k$ is the turn ratio of the auxiliary control winding and the main control winding. $L_{1}$ is the equivalent inductance of the main control winding in the working state of thyristor.

The conclusion can be drawn from the formula (9): the equivalent inductance of MCR can be adjusted smoothly by the thyristor conduction angle $\alpha$. When the reactor angle $\alpha$ is zero, the equivalent inductance of MCR is maximum, when the thyristor control angle becomes larger and 
larger, the equivalent inductance of MCR continuously decreases. It has characteristics of Auto-tuning inductance for the dynamic harmonic filter

\section{Engineering application example}

The dynamic harmonic filter to filter out the maximum $5^{\text {th }}$ harmonic current of $60 \mathrm{~A}$ has been developed by the author. It has been successfully applied to the Changxin South Cement Company Limited and Quyang Jinyu India Cements Limited and other users.

Measurement data of the power distribution room in 6\# kiln head of Changxin South Cement Company Limited. The RMS of current, fundamental current and $5^{\text {th }}$ harmonic current were measured using the power quality analyzer C.A.8335. The measurement data are shown in Figure 2 when the dynamic harmonic filter is switch OFF or ON.

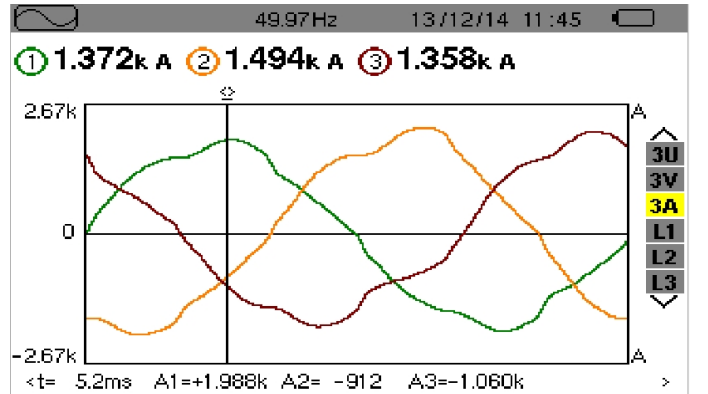

RMS

(a) RMS when switch OFF

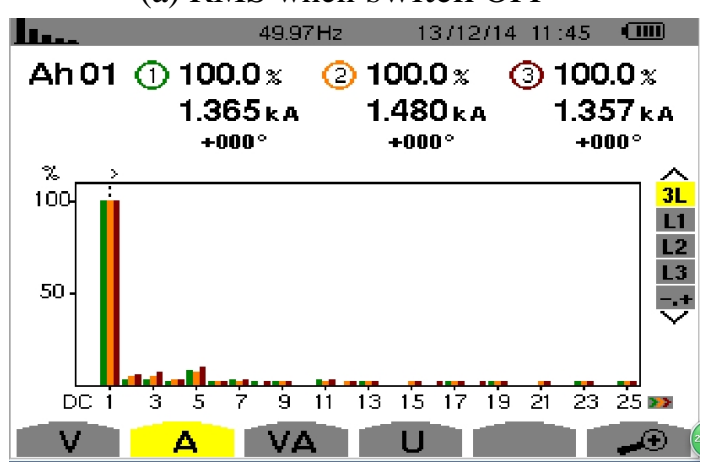

(c) Fundamental current when switch OFF
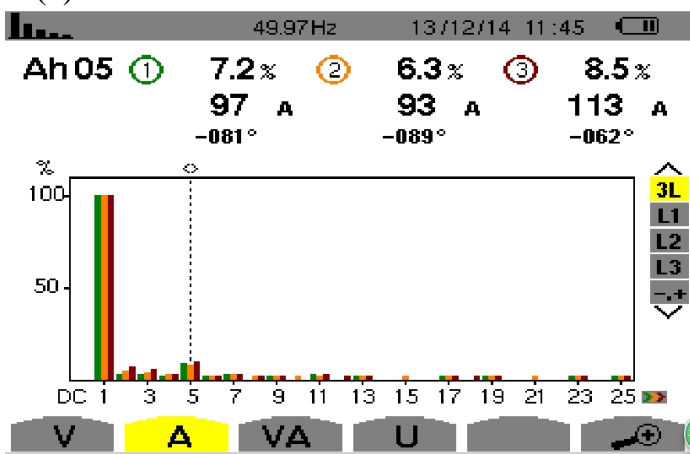

(e) $5^{\text {th }}$ harmonic current when switch OFF

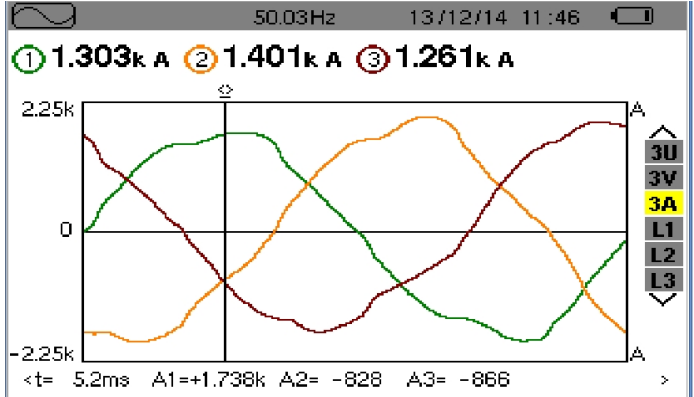

RMS THD CF t

(b) RMS when switch ON

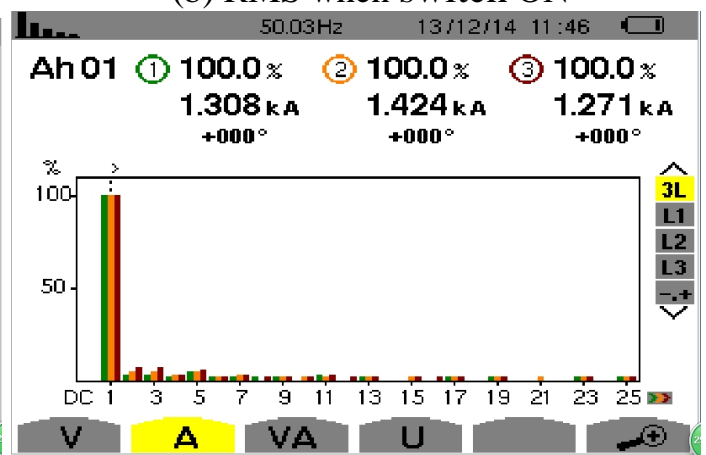

(b) Fundamental current when switch $\mathrm{ON}$

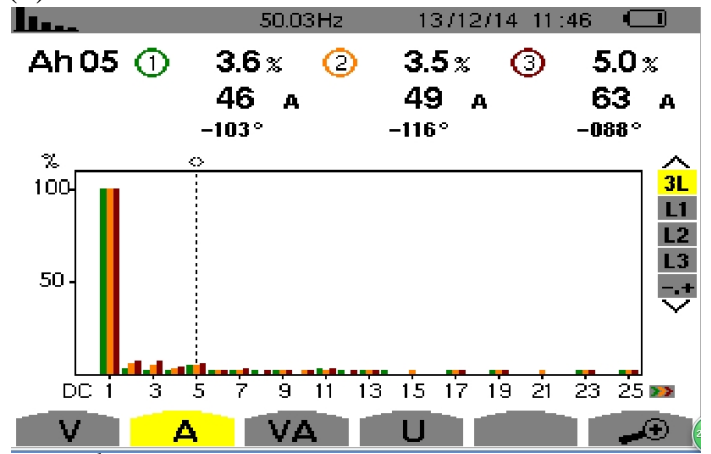

(f) $5^{\text {th }}$ harmonic current when switch ON

Fig. 2 Measurement data of the power distribution room in $6 \#$ kiln head

Data analysis. The following conclusions can be drawn from Fig. 2:

(1) the fundamental and 5th harmonic currents are reduced at the same time when the dynamic harmonic filter is in a state of operation $(\mathrm{ON})$;

(2) RMS of current: the 1372A (switch off) is reduced to $1302 \mathrm{~A}$ (switch on), reduced by $69 \mathrm{~A}$. So energy saving effect is $6 \% \sim 10 \%$;

(3) Fundamental current: 1365A (switch off)is reduced to 1308A(switch on), reduced by 58A. It reduce the 5\% 10\% fundamental current; 
(4) 5th harmonic current: 97A (switch off) is reduced to 46A (switch on), reduced by 51A.

\section{Conclusions}

The harmonic suppression and reactive power compensation must be needed to improve the electric energy quality and saving energy in our power industry. Firstly, the paper analyzes quantitatively the harmonic loss of the power equipment in power distribution system and the drift of the filter resonance point caused by the change of the capacitance of the capacitor. Secondly, discusses the energy saving mechanism of the dynamic harmonic filter. Lastly, advances the method for keeping filtering effect of the dynamic harmonic filter.

The dynamic harmonic filter to filter out the maximum $5^{\text {th }}$ harmonic current of $60 \mathrm{~A}$ has been developed by the author. It has been successfully applied to the Changxin South Cement Company Limited and Quyang Jinyu India Cements Limited and other users. The following results were achieved from application results:

(1) The dynamic harmonic filter has effectively suppressed the harmonic and improve the power quality, improved the service life of the electrical equipment, reduced the harmonic impact on the power grid, effectively controlled the harmonic pollution.

(2) The dynamic harmonic filter can reduce the 5\% 10\% fundamental current.

(3) The dynamic harmonic filter direct power saving effect is more than $5 \%$, and the copper loss and iron loss is reduced by $3 \%$. The total energy saved in the system reaches more than $10 \%$. It has the energy saving effect, enhances the power factor, the annual electricity saving benefit is obvious.

\section{Acknowledgements}

This work was financially supported by the projects of Hubei Science and Technology Plans of China (Granted No: 2014BAA011 and 2013BAA009).

\section{References}

[1] Lin Haixue: Chin.Soc.for Elec.Eng., Vol.34(2014), p. 5073

[2] Zhang Lisheng, Shi Jia, Guo Chao, Shi Dafa: J. of Power Technology J., Vol.38(2014) , p. 152

[3] Zhou Liangcail, Zhang Baohuil, etc.: Automation of Electric Power Systems, Vol.35(2011), p. 61

[4] Chen Jing, Lei Lei, Yuan Youxin, etc. : J. of Electrical Engineering, Vol.2(2013), p. 3

[5] Chang Yufang, Yuan youxin, etc.: J. of Wuhan University of Technology,Vol.33(2011), p. 127 